\title{
Change of grading, injective dimension and dualizing complexes
}

\author{
A. Solotar, P. Zadunaisky*
}

\begin{abstract}
Let $G, H$ be groups, $\varphi: G \longrightarrow H$ a group morphism, and $A$ a $G$-graded algebra. The morphism $\varphi$ induces an $H$-grading on $A$, and on any G-graded $A$ module, which thus becomes an $H$-graded $A$-module. Given an injective $G$-graded $A$-module, we give bounds for its injective dimension when seen as $H$-graded $A$ module. Following ideas by Van den Bergh, we give an application of our results to the stability of dualizing complexes through change of grading.
\end{abstract}

2010 MSC: 16D50, 16E10, 16E65, 16W50, 18G05.

Keywords: injective modules, change of grading, dualizing complexes.

\section{Introduction}

Graded rings are ubiquitous in algebra. One of the main reasons is that the presence of a grading simplifies proofs and allows to generalize many results (for example, the theories of commutative and noncommutative graded algebras are easier to reconcile than their ungraded counterparts). Furthermore, results can often be transfered from the graded to the ungraded context through standard techniques. In more categorical terms, there is a natural forgetful functor from the category $\mathrm{Gr}_{G} A$ of graded modules over a G-graded algebra $A$, to the category $\operatorname{Mod} A$ of modules over $A$, and the challenge is to find a way to transfer information in the opposite direction. When $G=\mathbb{Z}$ this is usually done through "filtered-and-graded" arguments and spectral sequences. In this article we exploit a different technique, namely the existence of three functors $\varphi_{!}, \varphi^{*}, \varphi_{*}$, where $\varphi_{!}: \operatorname{Gr}_{G} A \longrightarrow \operatorname{Mod} A$ is the usual forgetful functor (sometimes also called the push-down functor), $\varphi^{*}$ is its right adjoint, and $\varphi_{*}$ is the right adjoint of $\varphi^{*}$. This technique has two advantages over the usual filtered-and-graded methods, namely that it does not depend on the choice of a non-canonical filtration, and that the group $G$ is arbitrary. Its main drawback is that the functors in this triple do not

*This work has been supported by the projects UBACYT 20020130 100533BA, pip-conicet $11220150100483 \mathrm{CO}$, and MATHAMSUD-REPHOMOL. The first named author is a research member of CONICET (Argentina). The second named author is a FAPESP PostDoc Fellow, grant: 2016-25984-1 São Paulo Research Foundation (FAPESP). 
preserve finite generation, noetherianity, or other "finiteness" properties unless further hypotheses are in place.

The problem we consider is the following. Suppose you are given an injective object $I$ in the category $\mathrm{Gr}_{\mathbb{Z}} A$. In general $I$ is not injective as $A$-module, but if $A$ is noetherian then its injective dimension is at most one. Now, what happens if we consider gradings by more general groups? In general, given groups $G, H$ and a group morphism $\varphi: G \longrightarrow H$, any $G$-graded object can be seen as an $H$-graded object through $\varphi$, see paragraph 2.3. In particular a G-graded algebra $A$ inherits an $H$-grading, and there is a natural functor $\varphi_{!}: \operatorname{Gr}_{G} A \longrightarrow \mathrm{Gr}_{H} A$, between the categories of $G$-graded and $H$-graded modules. The question thus becomes: given an injective object $I$ in $\operatorname{Gr}_{G} A$, what is the injective dimension of $\varphi_{!}(I)$ in $\mathrm{Gr}_{H} A$ ?

This question has been considered several times in the literature, but it has received no unified treatment. A classical result of R. Fossum and H.-B. Foxby [FF74, Theorem 4.10] states that if $A$ is $\mathbb{Z}$-graded noetherian and commutative then a $\mathbb{Z}$-graded-injective module has injective dimension at most 1. M. Van den Bergh claims in the article [vdB97, below Definition 6.1] that this result extends to the noncommutative case if the algebra is $\mathbb{N}$-graded and $A_{0}$ is equal to the base field; a proof of this fact can be found in the preprint [Yek14]. Other antecedents include [Eks89], where it is shown that if $A$ is a noetherian $\mathbb{Z}$-graded algebra then the injective dimension of $A$ is finite if and only if its graded injective dimension is finite. Following the ideas of [Lev92, section 3], one can show that if $A$ is $\mathbb{N}$-graded and noetherian, and $M$ is a $\mathbb{Z}$-graded module such that $M_{n}=0$ for $n \ll 0$, then the graded injective dimension of $M$ coincides with its injective dimension as $A$-module. Most of these results are obtained by the usual route of going from ungraded to graded objects through filtrations and spectral sequences. The only result that we could find in the literature regarding injective modules graded by groups other than $\mathbb{Z}$ states that if $A$ is graded over a finite group then a graded module is graded injective if and only if it is injective [NVOo4, 2.5.2].

In order to give a general answer to the question we work with the functors $\varphi_{!}, \varphi^{*}, \varphi_{*}$ mentioned above, which were originally introduced by A. Polishchuk and L. Positselski in [PP12]. These functors, collectively called the change of grading functors, turn out to be particularly well-adapted to the transfer of information of homological nature. Our main result, which includes most of the previous ones as special cases, is the following.

Theorem. Let $\varphi: G \longrightarrow H$ be a group morphism, let $L=\operatorname{ker} \varphi$ and let $d$ be the projective dimension of the trivial L-module $\mathbb{k}$. Let $A$ be a G-graded noetherian algebra, and let $I$ be an injective object of $\mathrm{Gr}_{G} A$. Then the injective dimension of $\varphi_{!}(I)$ is at most $d$.

The proof depends on two facts. First, that if $I$ is G-graded injective then $\varphi_{!}(I)$ is an injective object in the additive subcategory generated by all modules of the form $\varphi_{!}(M)$ with $M$ a G-graded $A$-module; in other words, modules in the image of $\varphi$ ! are $\operatorname{Hom}_{A}^{H}\left(-, \varphi_{!}(I)\right)$-acyclic and hence can be used to build acyclic resolutions, see Lemma 3.5 . The second is a result of independent interest, stating that given an $\mathrm{H}$ graded $A$-module $N$ we can obtain a resolution of $N$ by objects in the additive category generated by $\varphi_{!}\left(\varphi^{*}(N)\right)$, see Proposition 3.4 this resolution can be used to calculate 
the $H$-graded extension modules between $N$ and $\varphi_{!}(I)$, which gives the desired bound.

The article is structured as follows. In Section 2 we review some basic facts on the category of graded modules and recall some general properties of the change of grading functors established in the article $\left[\mathrm{RZ}_{15}\right]$. In Section 3 we prove our main results on how regrading affects injective dimension. Finally in Section 4 we give similar results at the derived level and use them to study the behavior of dualizing complexes with respect to regradings, a question originally raised by Van den Bergh in [vdB97].

Throughout the article $\mathbb{k}$ is a commutative ring, and unadorned hom spaces and tensor products are always over $\mathbb{k}$. Also all modules over rings are left modules unless otherwise stated. The letters $G, H$ will always denote groups, and $\varphi: G \longrightarrow H$ will be a group morphism.

Acknowledgements: The authors would like to thank Mariano Suárez-Álvarez for a careful reading of a previous version of this article.

\section{The change of grading functors}

2.1. A $G$-graded $\mathbb{k}$-module is a $\mathbb{k}$-module $V$ with a fixed decomposition $V=\bigoplus_{g \in G} V_{g}$; we say that $v \in V$ is homogeneous of degree $g$ if $v \in V_{g}$, and $V_{g}$ is called the $g$ homogeneous component of $V$. We usually say graded instead of $G$-graded if $G$ is clear from the context.

Given two G-graded modules $V$ and $W$, their tensor product is also a G-graded module, where for each $g \in G$

$$
(V \otimes W)_{g}=\bigoplus_{g^{\prime} \in G} V_{g^{\prime}} \otimes W_{\left(g^{\prime}\right)^{-1} g}
$$

A map between graded $\mathbb{k}$-modules $f: V \longrightarrow W$ is said to be G-homogeneous, or simply homogeneous, if $f\left(V_{g}\right) \subset W_{g}$ for all $g \in G$. By definition, a homogeneous map $f: V \longrightarrow W$ induces maps $f_{g}: V_{g} \longrightarrow W_{g}$ for each $g \in G$, and $f=\bigoplus_{g \in G} f_{g}$; we refer to $f_{g}$ as the homogeneous component of degree $g$ of $f$. The support of a G-graded $\mathbb{k}$-module $V$ is supp $V=\left\{g \in G \mid V_{g} \neq 0\right\}$.

The category $\mathrm{Gr}_{G} \mathbb{k}$ has $G$-graded modules as objects and homogeneous $\mathbb{k}$-linear maps as morphisms. Kernels and cokernels of homogeneous maps between graded $\mathbb{k}$-modules are graded in a natural way, so a complex

$$
0 \longrightarrow V^{\prime} \longrightarrow V \longrightarrow V^{\prime \prime} \longrightarrow 0
$$

in $G_{G} \mathbb{k}$ is a short exact sequence if and only if it is a short exact sequence of $\mathbb{k}$ modules, or equivalently if for each $g \in G$ the sequence formed by taking $g$-homogeneous components is exact.

Given an object $V$ in $\operatorname{Gr}_{G} \mathbb{k}$ and $g \in G$, we denote by $V[g]$ the G-graded $\mathbb{k}$-module whose homogeneous component of degree $g^{\prime}$ is $V[g]_{g^{\prime}}=V_{g^{\prime} g}$. This gives a natural autoequivalence of $\mathrm{Gr}_{G} \mathbb{k}$. 
2.2. We now recall the general definitions regarding $G$-graded $\mathbb{k}$-algebras. The reader is referred to [NVOo4, Chapter 2] for proofs and details.

A $G$-graded $\mathbb{k}$-algebra is a $G$-graded $\mathbb{k}$-module $A$ which is also a $\mathbb{k}$-algebra, such that for all $g, g^{\prime} \in G$ and all $a \in A_{g}, a^{\prime} \in A_{g^{\prime}}$ we have $a a^{\prime} \in A_{g g^{\prime}}$. If $A$ is a G-graded algebra then its structural map $\rho: A \longrightarrow A \otimes \mathbb{k}[G]$ is defined as $a \in A_{g} \mapsto a \otimes g \in$ $A_{g} \otimes \mathbb{k}[G]_{g}$ for each $g \in G$; the fact that $A$ is a G-graded algebra implies that this is a morphism of algebras.

A $G$-graded $A$-module is an $A$-module $M$ which is also a $G$-graded $\mathbb{k}$-module such that for each $g, g^{\prime} \in G$ and all $a \in A_{g}, m \in M_{g^{\prime}}$ it happens that $a m \in M_{g g^{\prime}}$. Once again, we usually say graded instead of $G$-graded. We say that $A$ is graded left noetherian if every graded $A$-submodule of a finitely generated graded $A$-module is also finitely generated. If $G$ is a polycyclic-by-finite group then $A$ is graded noetherian if and only if it is noetherian [CQ88, Theorem 2.2].

We denote by $\operatorname{Gr}_{G} A$ the category whose objects are G-graded $A$-modules and whose morphisms are $G$-homogeneous $A$-linear maps. Notice that if $M$ is a graded $A$-module then the graded $\mathbb{k}$-module $M[g]$ is also a graded $A$-module, with the same underlying $A$-module structure, so shifting also induces an autoequivalence of $\mathrm{Gr}_{G} A$.

The category $\mathrm{Gr}_{G} A$ has arbitrary direct sums and products. The direct sum of graded modules is again graded in an obvious way, but this is not the case for direct products. Given a collection of graded $A$-modules $\left\{V^{i} \mid i \in I\right\}$, their direct product is the graded $A$-module whose homogeneous decomposition is given by

$$
\bigoplus_{g \in G} \prod_{i \in I} V_{g}^{i}
$$

In other words, the forgetful functor $\mathcal{O}: \mathrm{Gr}_{G} A \longrightarrow \operatorname{Mod} A$ preserves direct sums, but not direct products.

The category $\mathrm{Gr}_{G} A$ is a Grothendieck category with enough projective and injective objects. Given an object $M$ of $\operatorname{Gr}_{G} A$, we will denote by $\operatorname{pdim}_{A}^{G} M$ and injdim ${ }_{A}^{G} M$ its projective and injective dimensions, respectively. Given two graded $A$-modules $M, N$ we denote by $\operatorname{Hom}_{A}^{G}(M, N)$ the $\mathbb{k}$-module of all $G$-homogeneous $A$-linear morphisms from $M$ to $N$. Since $\mathrm{Gr}_{G} A$ has enough injectives, we can define for each $i \geq 0$ the $i$-th right derived functor of $\operatorname{Hom}_{A}^{G}$, which we denote by $\mathcal{R}^{i} \operatorname{Hom}_{A}^{G}$.

There is also an enriched homomorphism functor $\underline{\mathrm{Hom}}_{A}^{G}$, given by

$$
\underline{\operatorname{Hom}}_{A}^{G}(M, N)=\bigoplus_{g \in G} \operatorname{Hom}_{A}^{G}(M, N[g])
$$

which is a G-graded $\mathbb{k}$-submodule of $\operatorname{Hom}_{\mathbb{k}}(M, N)$. We denote its right derived functors by $\mathcal{R}^{i} \underline{\text { Hom }}_{A}^{G}$.

2.3. Let $A$ be a G-graded $\mathbb{k}$-algebra. As shown in [RZ15, Section 1.3], a group homomorphism $\varphi: G \longrightarrow H$ induces functors $\varphi_{!}, \varphi_{*}: \mathrm{Gr}_{G} A \longrightarrow \mathrm{Gr}_{H} A$ and $\varphi^{*}: \mathrm{Gr}_{H} A \longrightarrow$ $\operatorname{Gr}_{G} A$. We quickly review the construction for completeness. 
Let $V$ be a $G$-graded $\mathbb{k}$-module. We define $\varphi_{!}(V)$ to be the $H$-graded $\mathbb{k}$-module whose homogeneous component of degree $h \in H$ is given by

$$
\varphi_{!}(V)_{h}=\bigoplus_{\{g \in G \mid \varphi(g)=h\}} V_{g} .
$$

Analogously given a map $f: V \longrightarrow W$ between $G$-graded $\mathbb{k}$-modules, we define $\varphi_{!}(f)$ to be the $\mathbb{k}$-linear map whose homogeneous component of degree $h \in H$ is given by

$$
\varphi_{!}(f)_{h}=\bigoplus_{\{g \in G \mid \varphi(f)=h\}} f_{g} .
$$

Notice that $\varphi_{!}(V)$ has the same underlying $\mathbb{k}$-module as $V$. In particular, $\varphi_{!}(A)$ is an $H$-graded $\mathbb{k}$-algebra which is equal to $A$ as $\mathbb{k}$-algebra, and if $V$ is a G-graded $A$ module then $\varphi_{!}(V)$ is an $H$-graded $\varphi_{!}(A)$-module with the same underlying $A$-module structure. Since the action of $A$ remains unchanged, if $f$ is $A$-linear then so is $\varphi_{!}(f)$. This defines the functor $\varphi_{!}: \operatorname{Gr}_{G} A \longrightarrow \operatorname{Gr}_{H} \varphi_{!}(A)$. From now on we usually write $A$ instead of $\varphi_{!}(A)$ to lighten up the notation, since the context will make it clear whether we are considering it as a $G$-graded or as an $H$-graded algebra.

We define $\varphi_{*}(V)$ and $\varphi_{*}(f)$, to be the $H$-graded $\mathbb{k}$-module, and $H$-homogeneous map whose homogeneous components of degree $h \in H$ are given by

$$
\varphi_{*}(V)_{h}=\prod_{\{g \in G \mid \varphi(g)=h\}} V_{g}, \quad \varphi_{*}(f)_{h}=\prod_{\{g \in G \mid \varphi(f)=h\}} f_{g},
$$

respectively. If $V$ is also an $A$-module, we define the action of a homogeneous element $a \in A_{g^{\prime}}$ with $g^{\prime} \in G$ over an element $\left(v_{g}\right)_{g \in \varphi^{-1}(h)} \in \varphi_{*}(V)_{h}$ as $a\left(v_{g}\right)=\left(a v_{g}\right)$. With this action $\varphi_{*}(V)$ becomes an $H$-graded $A$-module, and we have defined the functor $\varphi_{*}: \mathrm{Gr}_{G} A \longrightarrow \mathrm{Gr}_{H} A$.

Now let $V^{\prime}, W^{\prime}$ be $H$-graded $\mathbb{k}$-modules and let $f^{\prime}: V^{\prime} \longrightarrow W^{\prime}$ be a homogeneous map. We set $\varphi^{*}\left(V^{\prime}\right) \subset V^{\prime} \otimes \mathbb{k}[G]$ to be the subspace generated by all elements of the form $v \otimes g$ with $v \in V^{\prime}$ homogeneous of degree $\varphi(g)$, and $\varphi^{*}(f)(v \otimes g)=f(v) \otimes g$. In other words, for each $g \in G$ the homogeneous components of $\varphi^{*}\left(V^{\prime}\right)$ and $\varphi\left(f^{\prime}\right)$ of degree $g$ are given by

$$
\varphi^{*}\left(V^{\prime}\right)_{g}=V_{\varphi(g)}^{\prime} \otimes \mathbb{k g}, \quad \quad f_{g}=f_{\varphi(g)} \otimes \mathrm{Id} .
$$

If $V^{\prime}$ is an $H$-graded $A$-module, then $V^{\prime} \otimes \mathbb{k}[G]$ is an $A \otimes \mathbb{k}[G]$-module, and it is an induced $A$-module through the structure map $\rho: A \longrightarrow A \otimes \mathbb{k}[G]$; it is immediate to check that with this action it becomes a G-graded $A$-module with $\left(V^{\prime} \otimes \mathbb{k}[G]\right)_{g}=$ $V^{\prime} \otimes \mathbb{k} g$ for each $g \in G$, and that $\varphi^{*}\left(V^{\prime}\right) \subset V^{\prime} \otimes \mathbb{k}[G]$ is a G-graded $A$-submodule. It is also easy to check that if $f^{\prime}$ is homogeneous and $A$-linear then so is $\varphi^{*}\left(f^{\prime}\right)$. Thus we have defined a functor $\varphi^{*}: \mathrm{Gr}_{H} A \longrightarrow \mathrm{Gr}_{G} A$. 
2.4. We refer to $\varphi_{!}, \varphi^{*}$ and $\varphi_{*}$ collectively as the change of grading functors. It is clear from the definitions that the change of grading functors are exact, and that $\varphi_{!}, \varphi_{*}$ reflect exactness, i.e. a complex is exact if and only if its image by any of them is also exact. The functor $\varphi^{*}$ reflects exactness if and only if $\varphi$ is surjective. As mentioned before, we have some adjointness relations between these functors.

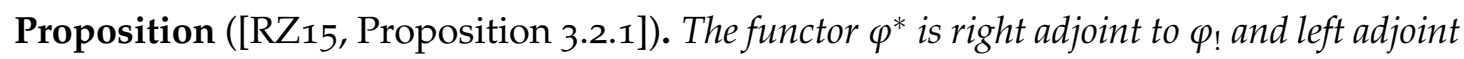
to $\varphi_{*}$.

Proof. Let $M$ be an object of $\mathrm{Gr}_{G} A$ and $N$ an object of $\mathrm{Gr}_{H} A$. We define maps

$$
\operatorname{Hom}_{A}^{H}(\varphi !(M), N) \underset{\beta}{\stackrel{\alpha}{\rightleftarrows}} \operatorname{Hom}_{A}^{G}\left(M, \varphi^{*}(N)\right)
$$

as follows. Given $f: \varphi_{!}(M) \longrightarrow N$, for each $g \in G$ and each $m \in M_{g}$ set $\alpha(f)(m)=$ $f(m) \otimes g$. Conversely, given $f: M \longrightarrow \varphi^{*}(N)$, let $\epsilon: \mathbb{k}[G] \longrightarrow \mathbb{k}$ be the counit of $\mathbb{k}[G]$, i.e. the algebra map defined by setting $\epsilon(g)=1$, and set $\beta(f)=1 \otimes \epsilon \circ f$. Direct computation shows that these maps are well defined, natural, and mutual inverses. Thus $\varphi_{!}$is the left adjoint of $\varphi^{*}$.

Now we define maps

$$
\operatorname{Hom}_{A}^{G}\left(\varphi^{*}(N), M\right) \underset{{ }_{\delta}}{\stackrel{\gamma}{\rightleftarrows}} \operatorname{Hom}_{A}^{H}\left(N, \varphi_{*}(M)\right)
$$

as follows. Given $f: \varphi^{*}(N) \longrightarrow M$, for each $h \in H$ and each $n \in N_{h}$ we set $\gamma(f)(n)=$ $(f(n \otimes g))_{g \in \varphi^{-1}(h)}$. Conversely, given $f: N \longrightarrow \varphi_{*}(M)$, for each $g \in G$ and $n \in N_{\varphi(g)}$ we have $f(n) \in \prod_{g^{\prime} \in \varphi^{-1}(h)} M_{g^{\prime}}$, so we can set $\delta(f)(n \otimes g)$ as the $g$-th component of $f(n)$. Once again direct computation shows that these maps are well defined, natural, and mutual inverses.

\section{Injective dimension and change of grading}

Recall that $G, H$ are groups and $\varphi: G \longrightarrow H$ is a group morphism. We set $L=\operatorname{ker} \varphi$. Throughout this section $A$ denotes a G-graded $\mathbb{k}$-algebra.

3.1. As stated in the Introduction, a G-graded $A$-module is projective if and only if it is projective as $A$-module, i.e. the functor $\varphi_{\text {! }}$ preserves the projective dimension of an object. Our aim is to describe how $\varphi_{\text {! }}$ affects the injective dimension of an object. We begin by recalling a previous result related to this problem.

Proposition ([RZ15. Corollaries 3.2.2, 3.2.3]). Let $M$ be an object of $\mathrm{Gr}_{G} A$. Then the following hold.

(a) $\operatorname{pdim}_{A}^{G} M=\operatorname{pdim}_{A}^{H} \varphi_{!}(M)$ and $\operatorname{injdim}_{A}^{G} M \leq \operatorname{injdim}_{A}^{H} \varphi_{!}(M)$.

(b) $\operatorname{pdim}_{A}^{G} M \leq \operatorname{pdim}_{A}^{H} \varphi_{*}(M)$ and $\operatorname{injdim}_{A}^{G} M=\operatorname{injdim}_{A}^{H} \varphi_{*}(M)$. 
3.2. The natural inclusion of the direct sum of a family into its product gives rise to a natural transformation $\eta: \varphi_{!} \Rightarrow \varphi_{*}$. Notice that $\eta(M): \varphi_{!}(M) \longrightarrow \varphi_{*}(M)$ is an isomorphism if and only if for each $h \in H$ the set $\operatorname{supp} M \cap \varphi^{-1}(h)$ is finite. If this happens we say that $M$ is $\varphi$-finite. The following theorem follows immediately from Proposition 3.1.

Theorem. If an object $M$ of $\operatorname{Gr}_{G} A$ is $\varphi$-finite then $\operatorname{injdim}_{A}^{G} M=\operatorname{injdim}_{A}^{H} \varphi_{!}(M)$.

Remark. If $|L|<\infty$ then every G-graded A-module is $\varphi$-finite. Also, if $A$ is $\varphi$-finite then every finitely generated G-graded A-module is $\varphi$-finite, so this result applies in many usual situations. For example, assume $A$ is $\mathbb{N}^{r}$-graded for some $r>0$, i.e. $A$ is $\mathbb{Z}^{r}$-graded and $A_{\xi}=0$ if $\xi \notin \mathbb{N}^{r}$. Let $\psi: \mathbb{Z}^{r} \longrightarrow \mathbb{Z}$ be the morphism $\psi\left(z_{1}, \ldots, z_{r}\right)=z_{1}+\cdots+z_{r}$. Then $\psi_{!}(A)$ is $\mathbb{Z}$-graded, and furthermore $A_{z}=0$ if $z \notin \mathbb{N}$. Since for each $z \in \mathbb{N}$ the set $\psi^{-1}(z) \cap \mathbb{N}^{r}$ is finite, the algebra $A$ is $\psi$-finite. Applying the theorem we see that $\operatorname{injdim}_{A}^{\mathbb{Z}^{r}} A=\operatorname{injdim}_{A}^{\mathbb{Z}} \psi_{!}(A)$. If $A$ is also noetherian then by [Lev92, 3.3 Lemma] we see that $\operatorname{injdim}_{A}^{\mathbb{Z}^{r}} A=\operatorname{injdim}_{A} A$.

3.3. The algebra $\mathbb{k}[G]$ is a G-graded $\mathbb{k}$-algebra, and hence through $\varphi$ it is also an $H$ graded algebra, so we may consider the category of $H$-graded $\mathbb{k}[G]$-modules $\mathrm{Gr}_{H} \mathbb{k}[G]$. The algebra $\mathbb{k}[H]$ is an object in this category with its usual $H$-grading and the action of $\mathbb{k}[G]$ induced by $\varphi$. By [Mon93, Theorem 8.5.6], the functor $-\otimes \mathbb{k}[H]: \operatorname{Mod} \mathbb{k}[L] \longrightarrow$ $\mathrm{Gr}_{H} \mathbb{k}[G]$ is an equivalence of categories. In particular the projective dimension of $\mathbb{k}[H]$ in $\mathrm{Gr}_{H} \mathbb{k}[G]$ equals pdim $\mathbb{k}_{\mathbb{k}}[L] \mathbb{k}$.

3.4. Given an object $N$ of $\mathrm{Gr}_{H} A$ we denote by $\mathcal{S}(N)$ the smallest subclass of objects of $\mathrm{Gr}_{H} A$ containing the set $\left\{\varphi_{!}\left(\varphi^{*}(N[h])\right) \mid h \in H\right\}$ and closed under direct sums and direct summands.

Proposition. Set $d=\operatorname{pdim}_{\mathbb{k}[G]}^{H} \mathbb{k}[H]=\operatorname{pdim}_{\mathbb{k}[L]} \mathbb{k}$. Every H-graded A-module $N$ has a resolution of length at most $d$ by objects of $\mathcal{S}(N)$.

Proof. We begin by defining a functor $D_{N}: \mathrm{Gr}_{H} \mathbb{k}[G] \longrightarrow \mathrm{Gr}_{H} A$. Given an object $V$ of $\mathrm{Gr}_{H} \mathbb{k}[G]$, the tensor product $N \otimes V$ is an $A$-module with action induced by the map $\rho: A \longrightarrow A \otimes \mathbb{k}[G]$, and we set $D_{N}(V)$ to be the $A$-submodule $\bigoplus_{h \in H} N_{h} \otimes V_{h}$, with the obvious $H$-grading. Given a morphism $f: V \longrightarrow W$ in $\operatorname{Gr}_{H} \mathbb{k}[G]$, we set $D_{N}(f)$ as the restriction and correstriction of $\operatorname{Id}_{N} \otimes f$.

Fix $h \in H$. By definition $D_{N}(\mathbb{k}[G][h])$ and $\varphi_{!}\left(\varphi^{*}\left(N\left[h^{-1}\right]\right)\right)[h]$ are $A$-submodules of $N \otimes \mathbb{k}[G]$, and it is immediate to check that in both cases the homogeneous component of degree $h^{\prime} \in H$ is $N_{h} \otimes \mathbb{k}[G]_{h h^{\prime}}$, so in fact these two $H$-graded $A$-modules are equal. Furthermore, if $P$ is any projective object in $\mathrm{Gr}_{H} \mathbb{k}[G]$ then there exists an object $Q$ such that $P \oplus Q$ is a free $H$-graded $\mathbb{k}[G]$-module, which is isomorphic to $\bigoplus_{i \in I}(\mathbb{k}[G])\left[h_{i}\right]$ for some index set $I$, not necessarily finite, with $h_{i} \in H$. Now $D_{N}$ commutes with direct summs, $D_{N}(P)$ is a direct summand of $D_{N}(P \oplus Q) \cong \bigoplus_{i \in I} D_{N}\left(\mathbb{k}[G]\left[h_{i}\right]\right)=$ $\bigoplus_{i \in I} \varphi_{!}\left(\varphi^{*}\left(N\left[h_{i}^{-1}\right]\right)\right)\left[h_{i}\right]$, which obviously lies in $\mathcal{S}(N)$.

For each $h \in H$ we define a map $n \in N_{h} \mapsto n \otimes h \in D_{N}(\mathbb{k}[H])_{h}$; the direct sum of these maps gives us an isomorphism $N \cong D_{N}(\mathbb{k}[H])$. Taking a projective resolution $P^{\bullet}$ of $\mathbb{k}[H]$ of length $d$ and applying $D_{N}$, we obtain a complex $D_{N}\left(P^{\bullet}\right) \longrightarrow D_{N}(\mathbb{k}[H]) \cong$ $N$; since $\mathbb{k}[G]$ is a free $\mathbb{k}$-module, projective $\mathbb{k}[G]$-modules are projective over $\mathbb{k}$ so this 
is an exact complex, and from the previous paragraph we see that it is a resolution of $N$ by objects in $\mathcal{S}(N)$.

3.5. Let $M$ be a G-graded $A$-module. Recall that $\varphi^{*}\left(\varphi_{!}(M)\right) \subset M \otimes \mathbb{k}[G]$ consists of all $m \otimes g^{\prime}$ with $m \in M_{g}$ and $\varphi(g)=\varphi\left(g^{\prime}\right)$. For each $l \in L$ we have a map $M[l] \longrightarrow \varphi^{*} \varphi_{!}(M)$ whose homogeneous component of degree $g \in G$ is given by $m \in M[l]_{g} \mapsto m \otimes g l \in \varphi^{*} \varphi_{!}(M)$. This induces a natural map $\bigoplus_{l \in L} M[l] \longrightarrow \varphi^{*} \varphi_{!}(M)$. This map has an inverse, given by $m \otimes g^{\prime} \in \varphi^{*}\left(\varphi_{!}(M)\right) \mapsto m \in M\left[g^{-1} g^{\prime}\right]$, so we get a natural isomorphism $\varphi^{*}\left(\varphi_{!}(M)\right) \cong \bigoplus_{l \in L} M[l]$. This observation is used in the following lemma.

Lemma. Assume A is left G-graded noetherian. Let I, $M$ be objects of $\mathrm{Gr}_{G} A$ with I injective, and let $N$ be a direct summand of $\varphi_{!}(M)$. Then $\mathcal{R}^{i} \operatorname{Hom}_{A}^{H}\left(N, \varphi_{!}(I)\right)=0$ for all $i>0$.

Proof. It is enough to show that the result holds for $N=\varphi_{!}(M)$. In that case we have isomorphisms

$$
\operatorname{Hom}_{A}^{H}\left(\varphi_{!}(M), \varphi_{!}(I)\right) \cong \operatorname{Hom}_{A}^{G}\left(M, \varphi^{*}\left(\varphi_{!}(I)\right)\right) \cong \operatorname{Hom}_{A}^{G}\left(M, \bigoplus_{l \in L} I[l]\right)
$$

Since this isomorphism is natural in the first variable, we obtain for each $i \geq 0$ an isomorphism

$$
\mathcal{R}^{i} \operatorname{Hom}_{A}^{H}\left(\varphi_{!}(M), \varphi_{!}(I)\right) \cong \mathcal{R}^{i} \operatorname{Hom}_{A}^{G}\left(M, \bigoplus_{l \in L} I[l]\right) .
$$

Now by the graded version of the Bass-Papp Theorem (see [GWo4, Theorem 5.23] for a proof in the ungraded case, which adapts easily to the graded context), the fact that $A$ is left G-graded noetherian implies that $\bigoplus_{l \in L} I[l]$ is injective, and hence the last isomorphism implies $\mathcal{R}^{i} \operatorname{Hom}_{A}^{H}\left(\varphi_{!}(M), \varphi_{!}(I)\right)=0$.

Remark. We point out that the proof does not use the full Bass-Papp Theorem, just the fact that the direct sum of an arbitrary family of shifted copies of the same injective module is again injective, so we may wonder whether this property is weaker than G-graded noetherianity. In the ungraded case a module is called $\Sigma$-injective if the direct sum of arbitrarily many copies of it is injective. Say that a G-graded A-module is graded $\Sigma$-injective if an arbitrary direct sum of shifted copies of itself is injective. Then by a reasoning analogous to that of [FW67. Theorem, pp. 205-6] one can prove that an algebra is left G-graded noetherian if and only if every injective object of $\operatorname{Gr}_{G} A$ is graded $\Sigma$-injective. We thank MathOverflow user Fred Rohrer for the reference.

3.6. We are now ready to prove the main result of this section.

Theorem. Set $d=\operatorname{pdim}_{\mathbb{k}[L]} \mathbb{k}$. Assume $A$ is left G-graded noetherian. For every object $M$ of $\operatorname{Gr}_{G} A$ we have injdim ${ }_{A}^{G} M \leq \operatorname{injdim}_{A}^{H} \varphi_{!}(M) \leq \operatorname{injdim}_{A}^{G} M+d$

Proof. The first inequality holds by Proposition 3.1. The case where $M$ is of infinite injective dimension is trivially true, so let us consider the case where $n=\operatorname{injdim}_{A}^{G} M$ is finite. In this case we work by induction. 
If $n=0$ then $M$ is injective in $\operatorname{Gr}_{G} A$. Let $N$ be an object of $\mathrm{Gr}_{H} A$, and let $P^{\bullet} \longrightarrow N$ be a resolution of $N$ of length $d$ by objects of $\mathcal{S}(N)$ as in Proposition 3.4 It follows from Lemma $3 \cdot 5$ that $\mathcal{R}^{i} \operatorname{Hom}_{A}^{H}\left(P, \varphi_{!}(I)\right)=0$ for every object $P$ of $\mathcal{S}(N)$, so in fact $P^{\bullet}$ is an acyclic resolution of $N$ and

$$
\mathcal{R}^{i} \operatorname{Hom}_{A}^{H}\left(N, \varphi_{!}(M)\right) \cong H^{i}\left(\operatorname{Hom}_{A}^{H}\left(P^{\bullet}, \varphi_{!}(M)\right)\right)
$$

for each $i \geq 0$. Thus $\mathcal{R}^{i} \operatorname{Hom}_{A}^{H}\left(N, \varphi_{!}(M)\right)=0$ for all $i>d$, and since $N$ was arbitrary this implies that injdim ${ }_{A}^{H} \varphi_{!}(M) \leq d$.

Now assume that the result holds for all objects of $\operatorname{Gr}_{G} A$ with injective dimension less than $n$. Let $M \longrightarrow I$ be an injective envelope of $M$ in $\operatorname{Gr}_{G} A$, and let $M^{\prime}$ be its cokernel. Then $\operatorname{injdim}_{A}^{G} M^{\prime}=n-1$, and so by the inductive hypothesis $\operatorname{injdim}{ }_{A}^{H} \varphi_{!}\left(M^{\prime}\right) \leq n-1+d$. Now we have an exact sequence in $\mathrm{Gr}_{H} A$ of the form

$$
0 \longrightarrow \varphi_{!}(M) \longrightarrow \varphi_{!}(I) \longrightarrow \varphi_{!}\left(M^{\prime}\right) \longrightarrow 0 .
$$

By standard homological algebra the injective dimension of $\varphi_{!}(M)$ is bounded above by the maximum between injdim ${ }_{A}^{H} \varphi_{!}(I)+1 \leq d+1$ and $\operatorname{injdim}_{A}^{H} \varphi_{!}\left(M^{\prime}\right)+1 \leq n+d$. This gives us the desired inequality.

\section{Change of grading at the derived level and dualizing com- plexes}

Dualizing complexes for noncommutative rings were introduced by A. Yekutieli in the context of connected $\mathbb{N}$-graded algebras in order to study their local cohomology; they have proven to be very useful in the study of ring theoretical properties of non commutative rings, see for example [Yek92, Jør97, vdB97, YZ99, WZ03, YZo9], etc. A dualizing complex is essentially an object $R^{\bullet}$ in the derived category of $\operatorname{Mod} A^{e}$ such that the functor $\mathcal{R} \operatorname{Hom}_{A}\left(-, R^{\bullet}\right)$ is a duality between $\mathcal{D}^{b}(\operatorname{Mod} A)$ and $\mathcal{D}^{b}\left(\operatorname{Mod} A^{\text {op }}\right)$, for a precise definition see Definition 4.5. A graded dualizing complex in principle only guarantees dualities at the graded level, but according to Van den Bergh, a $\mathbb{Z}$-graded dualizing complex is also an ungraded dualizing complex [vdB97]. In this section we show that in fact a $\mathbb{Z}^{r}$-graded dualizing complex remains a dualizing complex after regrading. Once you have Theorem 3.6, the proof in the $\mathbb{Z}^{r}$-graded case is no more difficult than in the $\mathbb{Z}$-graded case, except for the technical complications due to the extra gradings. Still, we felt it was worthwhile to develop these technicalities in order to obtain a precise statement of Theorem 4.8 .

Throughout this section $\mathbb{k}$ is a field, $G$ is an abelian group, and $A$ is a G-graded $\mathbb{k}$-algebra. We denote by $A^{e}$ the enveloping algebra $A \otimes A^{\mathrm{op}}$; since $G$ is abelian both $A^{\mathrm{op}}$ and $A^{e}$ are G-graded algebras.

4.1. Let us fix some notation regarding derived categories. Given an abelian category $\mathcal{A}$, we denote by $\mathcal{K}(A)$ the category of complexes of objects of $\mathcal{A}$ with homotopy classes of maps of complexes as morphisms, and by $\mathcal{D}(\mathcal{A})$ the derived category of 
$\mathcal{A}$. As usual we denote by $\mathcal{D}^{+}(\mathcal{A}), \mathcal{D}^{-}(\mathcal{A}), \mathcal{D}^{b}(\mathcal{A})$ the full subcategories of $\mathcal{D}(A)$ consisting of left bounded, right bounded and bounded complexes. Recall that an injective resolution of a left bounded complex $R^{\bullet}$ is a quasi-isomorphism $R^{\bullet} \longrightarrow I^{\bullet}$ where $I^{\bullet}$ is a left bounded complex formed by injective objects of $\mathcal{A}$. If $\mathcal{A}$ has enough injectives then every left bounded complex has an injective resolution. Analogous remarks apply for projective resolutions of right bounded complexes.

If $F: \mathcal{A} \longrightarrow \mathcal{B}$ is an exact functor between abelian categories, then by the universal property of derived categories there is an induced functor $\mathcal{D}(\mathcal{A}) \longrightarrow \mathcal{D}(\mathcal{B})$, which by abuse of notation we will also denote by $F$.

4.2. The maps $a \in A \mapsto a \otimes 1 \in A^{e}$ and $a \in A^{\mathrm{op}} \mapsto 1 \otimes a \in A^{e}$ induce restriction functors $\operatorname{Res}_{A}: \operatorname{Gr}_{G} A^{e} \longrightarrow \mathrm{Gr}_{G} A$ and $\operatorname{Res}_{A}$ op $: \operatorname{Gr}_{G} A^{e} \longrightarrow \mathrm{Gr}_{G} A^{\text {op }}$. These functors are exact and preserve projectives and injectives, which can be proved following the lines of the proof in the case $G=\mathbb{Z}$ found in [Yek92, Lemma 2.1]. If $H$ is any group and $\varphi: G \longrightarrow H$ is a group morphism then it is clear that the associated change of grading functors commute with the restriction functors in the obvious sense. Since restriction and change of grading functors are exact, they induce exact functors between the corresponding derived categories.

4.3. There exists a functor

$$
\underline{\mathrm{Hom}}_{A}^{G}: \mathcal{K}\left(\mathrm{Gr}_{G} A^{e}\right)^{\mathrm{op}} \times \mathcal{K}\left(\operatorname{Gr}_{G} A^{e}\right) \longrightarrow \mathcal{K}\left(\mathrm{Gr}_{G} A^{e}\right)
$$

defined as follows. Given complexes $M^{\bullet}, N^{\bullet}$, for each $n \in \mathbb{Z}$ we set

$$
\underline{\operatorname{Hom}}_{A}^{G}\left(N^{\bullet}, M^{\bullet}\right)^{n}=\prod_{p \in \mathbb{Z}} \underline{\operatorname{Hom}}_{A}^{G}\left(N^{p}, M^{p+n}\right),
$$

where the product is taken in the category of G-graded $A^{e}$-modules; this sequence of $G$-graded $A^{e}$-modules is made into a complex with differential

$$
d^{n}=\prod_{p \in \mathbb{Z}}\left((-1)^{n+1} \underline{\operatorname{Hom}}_{A}^{G}\left(d_{N}^{p}, M^{p+n}\right)+\underline{\operatorname{Hom}}_{A}^{G}\left(N^{p}, d_{M}^{p+n}\right)\right) .
$$

The action of $\underline{\operatorname{Hom}}_{A}^{G}$ on maps is defined in the usual way.

The functor $\underline{\mathrm{Hom}}_{A}^{G}$ has a right derived functor

$$
\mathcal{R} \underline{\mathrm{Hom}}_{A}^{G}: \mathcal{D}\left(\operatorname{Gr}_{G} A^{e}\right)^{\mathrm{op}} \times \mathcal{D}\left(\operatorname{Gr}_{G} A^{e}\right) \longrightarrow \mathcal{D}\left(\mathrm{Gr}_{G} A^{e}\right) .
$$

When $M^{\bullet}$ is an object of $\mathcal{D}^{+}\left(\mathrm{Gr}_{G} A^{e}\right)$ such that $M^{i}$ is injective as left $A$-module for each $i \in \mathbb{Z}$, then

$$
\mathcal{R} \underline{\operatorname{Hom}}_{A}^{G}\left(N^{\bullet}, M^{\bullet}\right) \cong \underline{\operatorname{Hom}}_{A}^{G}\left(N^{\bullet}, M^{\bullet}\right)
$$

for every object $N^{\bullet}$ of $\mathcal{D}\left(\operatorname{Gr}_{G} A^{e}\right)$. Analogously, if $N^{\bullet}$ is an object of $\mathcal{D}^{-}\left(\operatorname{Gr}_{G} A^{e}\right)$ such that $N^{i}$ is projective as left $A$-module for each $i \in \mathbb{Z}$, then

$$
\mathcal{R} \underline{\operatorname{Hom}}_{A}^{G}\left(N^{\bullet}, M^{\bullet}\right) \cong \underline{\operatorname{Hom}}_{A}^{G}\left(N^{\bullet}, M^{\bullet}\right)
$$

for every object $M^{\bullet}$ of $\mathcal{D}\left(\mathrm{Gr}_{G} A^{e}\right)$. This is proved in the case $G=\mathbb{Z}$ in [Yek92, Theorem 2.2], and the general proof follows the same reasoning. There is a completely analogous functor $\underline{\operatorname{Hom}}_{A}^{G}$ op whose derived functor $\mathcal{R} \underline{\operatorname{Hom}}_{A}^{G}$ op has similar properties. 
4.4. Let $R^{\bullet}$ be a complex of $A^{e}$-modules. Seeing $A^{\text {op }}$ as a complex of $A^{e}$-modules concentrated in homological degree 0 , there is a map $A^{\mathrm{op}} \longrightarrow \underline{\operatorname{Hom}}_{A}^{G}\left(R^{\bullet}, R^{\bullet}\right)$ given by sending $a \in A^{\text {op }}$ to right multiplication by $a$ acting on $R^{\bullet}$. Now let $P^{\bullet} \longrightarrow R^{\bullet}$ be a projective resolution of $R^{\bullet}$, so there is an isomorphism

$$
\mathcal{R} \underline{\operatorname{Hom}}_{A}^{G}\left(R^{\bullet}, R^{\bullet}\right) \cong \underline{\operatorname{Hom}}_{A}^{G}\left(P^{\bullet}, P^{\bullet}\right),
$$

and we get a map nat $A: A^{\text {op }} \longrightarrow \mathcal{R} \underline{\operatorname{Hom}}_{A}^{\mathbb{Z}^{r}}\left(R^{\bullet}, R^{\bullet}\right)$. This map is independent of the projective resolution we choose, so we refer to it as the natural map from $A^{\mathrm{op}}$ to $\mathcal{R} \underline{\operatorname{Hom}}_{A}^{\mathbb{Z}^{r}}\left(R^{\bullet}, R^{\bullet}\right)$. In the same way there is a natural map from $A$ to $\mathcal{R} \underline{\operatorname{Hom}}_{A^{\text {op }}}^{\mathbb{Z}^{r}}\left(R^{\bullet}, R^{\bullet}\right)$. The proof that these maps are independent of the chosen resolution is quite tedious but elementary; the reader is referred to [Zad14, Appendix $\mathrm{A}]$ for details.

4.5. Assume that $G=\mathbb{Z}^{r}$ for some $r \geq 0$. We say that $A$ is $\mathbb{N}^{r}$-graded if $\operatorname{supp} A \subset \mathbb{N}^{r}$, and that it is connected if $A_{0}=\mathbb{k}$. If $A$ is $\mathbb{N}^{r}$-graded then so are $A^{\mathrm{op}}$ and $A^{e}$, and they are connected if and only if $A$ is connected.

The following definition is adapted from [Yek92, Definition 3.3].

Definition. Let $A$ be a connected $\mathbb{N}^{r}$-graded noetherian algebra. $A \mathbb{Z}^{r}$-graded dualizing complex over $A$ is a bounded complex $R^{\bullet}$ of $A^{e}$-modules with the following properties.

(a) The cohomology modules of $\operatorname{Res}_{A}\left(R^{\bullet}\right)$ and $\operatorname{Res}_{A^{\text {op }}}\left(R^{\bullet}\right)$ are finitely generated.

(b) Both $\operatorname{Res}_{A}\left(R^{\bullet}\right)$ and $\operatorname{Res}_{A^{\text {op }}}\left(R^{\bullet}\right)$ have finite injective dimension.

(c) The maps nat $A: A^{\text {op }} \longrightarrow \mathcal{R} \underline{\operatorname{Hom}}_{A}^{\mathbb{Z}^{r}}\left(R^{\bullet}, R^{\bullet}\right)$ and nat $_{A^{\text {op }}}: A \longrightarrow \mathcal{R} \underline{\operatorname{Hom}}_{A^{\text {op }}}^{\mathbb{Z}^{r}}\left(R^{\bullet}, R^{\bullet}\right)$ are isomorphisms in $\mathcal{D}\left(\mathrm{Gr}_{\mathbb{Z}^{r}} A^{e}\right)$.

A dualizing complex in the ungraded sense is an object of $\mathcal{D}\left(\operatorname{Mod} A^{e}\right)$ which complies with the ungraded analogue of the previous definition. Our objective is to show that a $\mathbb{Z}^{r}$-graded dualizing complex remains a dualizing complex if we change (or forget) the grading. Since being finitely generated is independent of grading, item (a) of the definition remains true if we change or forget the grading. To see how item (b) behaves with respect to change of grading requires a derived version of Theorem 3.6. while item (c) is also invariant by change of grading by a simple argument. We provide the details in the following lemmas, in a slightly more general context.

4.6. Recall that given a group morphism $\varphi: G \longrightarrow H$, a $G$-graded $\mathbb{k}$-vector space $M$ is said to be $\varphi$-finite if supp $M \cap \varphi^{-1}(h)$ is a finite set for each $h \in H$.

Lemma. Let $\varphi: G \longrightarrow H$ be a group morphism and set $L=\operatorname{ker} \varphi$. Let $R^{\bullet}$ be a bounded complex of G-graded A-modules.

(a) If the cohomology modules of $R^{\bullet}$ are $\varphi$-finite then $\operatorname{injdim}_{A}^{G} R^{\bullet}=\operatorname{injdim}_{A}^{H} \varphi_{!}\left(R^{\bullet}\right)$

(b) Let $d=\operatorname{pdim}_{\mathbb{k}[L]} \mathbb{k}$. If $A$ is left $G$-graded noetherian then the following inequalities hold

$$
\operatorname{injdim}_{A}^{G} R^{\bullet} \leq \operatorname{injdim}_{A}^{H} \varphi_{!}\left(R^{\bullet}\right) \leq \operatorname{injdim}_{A}^{G} R^{\bullet}+d .
$$


Proof. Let $R^{\bullet} \longrightarrow I^{\bullet}$ be an injective resolution of minimal length. It is enough to prove the statement with $I^{\bullet}$ instead of $R^{\bullet}$.

Suppose $I^{\bullet}$ has $\varphi$-finite cohomology modules. Recall that there is a natural transformation $\eta: \varphi_{!} \Rightarrow \varphi_{*}$, and that $\eta(M)$ is an isomorphism if an only if $M$ is $\varphi$ finite. The class of $\varphi$-finite $G$-graded $A$-modules is closed by extensions, so applying [Har66, Proposition 7.1] (in the reference "thick" stands for "closed by extensions") we get that the map $\varphi_{!}\left(I^{\bullet}\right) \longrightarrow \varphi_{*}\left(I^{\bullet}\right)$ is a quasi-isomorphism, and since $\varphi_{*}$ preserves injectives it is an injective resolution, so injdim ${ }_{A}^{G} R^{\bullet} \geq \operatorname{injdim}_{A}^{H} \varphi_{!}\left(R^{\bullet}\right)$. If the inequality were strict, then we could truncate $\varphi_{*}\left(I^{\bullet}\right)$ to obtain a shorter complex of the form

$$
\cdots \longrightarrow \varphi_{*}\left(I^{j-1}\right) \longrightarrow \varphi_{*}\left(I^{j}\right) \longrightarrow \varphi_{*}\left(\text { coker } d^{j}\right) \longrightarrow 0 \longrightarrow \cdots
$$

with $\varphi_{*}\left(\right.$ coker $\left.d^{j}\right)$ an injective $H$-graded $A$-module. Since $\varphi_{*}$ preserves injective dimension by Proposition 3.1. this would contradict the fact that $I^{\bullet}$ is a minimal resolution of $R^{\bullet}$, so in fact injdim $R_{A}^{\bullet}=\operatorname{injdim}_{A}^{H} \varphi_{!}\left(R^{\bullet}\right)$. This proves item (a)

For item (b), assume first that $I^{\bullet}$ is bounded. We proceed by induction on $s$, the length of $I^{\bullet}$. The case $s=0$ is a special case of Theorem 3.6. Now let $t \in \mathbb{Z}$ be the minimal homological degree such that $I^{t} \neq 0$, and consider the exact sequence of complexes

$$
0 \longrightarrow I^{>t} \longrightarrow I^{\bullet} \longrightarrow I^{t} \longrightarrow 0,
$$

where $I^{t}$ is seen as a complex concentrated in homological degree $t$ and $I^{>t}$ is the subcomplex of $I^{\bullet}$ formed by all components in homological degree larger than $t$. Thus there is a distinguished triangle $\varphi_{!}\left(I^{>t}\right) \longrightarrow \varphi_{!}\left(I^{\bullet}\right) \longrightarrow \varphi_{!}\left(I^{t}\right) \longrightarrow$ in $\mathcal{D}\left(\operatorname{Gr}_{H} A\right)$. By the inductive hypothesis the inequality holds for the first and third complexes of the triangle, so a simple argument with long exact sequences shows that the corresponding inequality holds for $\varphi_{!}\left(I^{\bullet}\right)$.

Finally, if $I^{\bullet}$ is not bounded then we only have to prove that $\varphi_{!}\left(I^{\bullet}\right)$ does not have finite injective dimension. Now $\varphi^{*}$ preserves injective dimensions, and since $\varphi^{*}\left(\varphi_{!}\left(I^{\bullet}\right)\right) \cong \bigoplus_{l \in L} I[l]^{\bullet}$ has infinite injective dimension, so does $\varphi_{!}\left(I^{\bullet}\right)$.

4.7 Lemma. Let $G, H$ be abelian groups and $\varphi: G \longrightarrow H$ a group morphism. Assume $A$ is G-graded noetherian. Let $S^{\bullet}, R^{\bullet}$ be bounded complexes of G-graded $A^{e}$-modules such that the cohomology modules of $R^{\bullet}$ are finitely generated as left $A$-modules.

(a) The map

$$
\varphi_{!}\left(\mathcal{R} \underline{\operatorname{Hom}}_{A}^{G}\left(R^{\bullet}, S^{\bullet}\right)\right) \longrightarrow \mathcal{R} \underline{\operatorname{Hom}}_{A}^{H}\left(\varphi_{!}\left(R^{\bullet}\right), \varphi_{!}\left(S^{\bullet}\right)\right)
$$

is an isomorphism.

(b) The composition

$$
\begin{aligned}
\varphi_{!}(A) \stackrel{\varphi_{!}}{\stackrel{\left.\operatorname{nat}_{A}\right)}{\longrightarrow}} \varphi_{!}\left(\mathcal{R} \underline{\operatorname{Hom}}_{A}^{G}\left(R^{\bullet}, R^{\bullet}\right)\right) \longrightarrow \mathcal{R} \underline{\operatorname{Hom}_{A}^{H}}\left(\varphi_{!}\left(R^{\bullet}\right), \varphi_{!}\left(R^{\bullet}\right)\right) \\
\text { equals nat } \varphi_{!}(A): \varphi_{!}(A) \longrightarrow \mathcal{R} \operatorname{Hom}_{A}^{H}\left(\varphi_{!}\left(R^{\bullet}\right), \varphi_{!}\left(R^{\bullet}\right)\right)
\end{aligned}
$$


Proof. The map from item (a) is obtained as follows. Let $P^{\bullet} \longrightarrow R^{\bullet}$ be a projective resolution. Then $\varphi_{!}\left(P^{\bullet}\right) \longrightarrow \varphi_{!}\left(R^{\bullet}\right)$ is also a projective resolution since $\varphi_{!}$is exact and preserves projectives. Now by definition of $\underline{\operatorname{Hom}}_{A}^{G}\left(R^{\bullet}, S^{\bullet}\right)$, we have $\varphi_{!}\left(\underline{\operatorname{Hom}}_{A}^{G}\left(P^{\bullet}, S^{\bullet}\right)\right) \subset$ $\underline{\operatorname{Hom}}_{A}^{H}\left(\varphi_{!}\left(P^{\bullet}\right), \varphi_{!}\left(S^{\bullet}\right)\right)$, and the desired map is the inclusion. Once again this map is independent of the chosen projective resolution. Clearly item (b) follows from this.

If $R^{\bullet}$ and $S^{\bullet}$ are concentrated in homological degree 0 , item(a) is a well-known result, see for example [RZ15, Proposition 1.3.7]. The general result follows by standard arguments using [Har66, Proposition I.7.1(i)].

4.8. We are now ready to prove the main result of this section.

Theorem. Let $A$ be a connected $\mathbb{N}^{r}$-graded noetherian $\mathbb{k}$-algebra and let $R^{\bullet}$ be a $\mathbb{Z}^{r}$-graded dualizing complex over A.

(a) Let $s>0$ and let $\varphi: \mathbb{Z}^{r} \longrightarrow \mathbb{Z}^{s}$ be a group morphism such that $\varphi_{!}(A)$ is $\mathbb{N}^{s}$-graded connected. Then $\varphi_{!}\left(R^{\bullet}\right)$ is a $\mathbb{Z}^{s}$-graded dualizing complex over $\varphi_{!}(A)$ of injective dimension $\operatorname{inj\operatorname {dim}} \mathbb{Z}_{A}^{r} R^{\bullet}$.

(b) Let $\mathcal{O}: \mathcal{D}\left(\mathrm{Gr}_{\mathbb{Z}^{r}} A^{e}\right) \longrightarrow \mathcal{D}\left(\operatorname{Mod} A^{e}\right)$ be the forgetful functor. Then $\mathcal{O}\left(R^{\bullet}\right)$ is a dualizing complex over $A$ in the ungraded sense, of injective dimension at most injdim $\mathbb{Z}_{A}^{r} R^{\bullet}+$ 1.

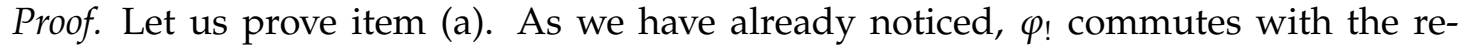
striction functors and does not change the fact that a bimodule is finitely generated as left or right $A$-module, so $\varphi_{!}\left(R^{\bullet}\right)$ complies with item (a) of Definition 4.5 . Since $A$ is $\mathbb{Z}^{r}$-graded noetherian it is also $\mathbb{Z}^{S}$-graded noetherian, and hence $\varphi_{!}(A)$ is locally finite; this implies that $A$ is $\varphi$-finite, otherwise $\varphi_{!}(A)$ would have a homogeneous component of infinite dimension. Since the cohomology modules of $R^{\bullet}$ are finitely generated, they are also $\varphi$-finite and hence by item (a) of Lemma $4.6 \operatorname{injdim}_{A}^{\mathbb{Z}^{s}} \varphi_{!}\left(R^{\bullet}\right)=\operatorname{injdim}_{A}^{\mathbb{Z}^{r}} R^{\bullet}$, so item (b) of Definition 4.5 also holds for $R^{\bullet}$. Finally item (c) of the definition follows immediately from item (b) of Lemma 4.7

We now prove item (b), Let $\psi: \mathbb{Z}^{r} \longrightarrow \mathbb{Z}$ be the map $\psi\left(z_{1}, \ldots, z_{r}\right)=z_{1}+\cdots+z_{r}$. Then $A$ is $\psi$-finite and $\psi_{!}(A)$ is connected $\mathbb{N}$-graded, so by the first item $\psi_{!}\left(R^{\bullet}\right)$ is a $\mathbb{Z}$-graded dualizing complex over $A$ of injective dimension injdim $\mathbb{Z}_{A}^{r} R^{\bullet}$. Now a similar reasoning as the one we used for the first item, but this time using item (b) of Lemma 4.6. shows that $\mathcal{O}\left(\psi_{!}\left(R^{\bullet}\right)\right)=\mathcal{O}\left(R^{\bullet}\right)$ is a dualizing complex and gives the bound for its injective dimension.

\section{References}

[CQ88] W. Chin and D. Quinn, Rings graded by polycyclic-by-finite groups, Proc. Amer. Math. Soc. 102 (1988), no. 2, 235-241.

[Eks89] E. K. Ekström, The Auslander condition on graded and filtered Noetherian rings, Année (Paris, 1987/1988), Lecture Notes in Math., vol. 1404, Springer, Berlin, 1989, pp. 220-245.

[FW67] C. Faith and E. A. Walker, Direct-sum representations of injective modules, J. Algebra 5 (1967), 203-221. 
[FF74] R. Fossum and H.-B. Foxby, The category of graded modules, Math. Scand. 35 (1974), 288-300.

[GWo4] K. R. Goodearl and R. B. Warfield Jr., An introduction to noncommutative Noetherian rings, 2nd ed., London Mathematical Society Student Texts, vol. 61, Cambridge University Press, Cambridge, 2004 .

[Har66] R. Hartshorne, Residues and duality, Lecture notes of a seminar on the work of A. Grothendieck, given at Harvard 1963/64. With an appendix by P. Deligne. Lecture Notes in Mathematics, No. 20, Springer-Verlag, Berlin, 1966.

[Jør97] P. Jørgensen, Local cohomology for non-commutative graded algebras, Comm. Algebra 25 (1997), no. 2, 575-591.

[Lev92] T. Levasseur, Some properties of noncommutative regular graded rings, Glasgow Math. J. 34 (1992), no. 3, 277-300.

[Mon93] S. Montgomery, Hopf algebras and their actions on rings, CBMS Regional Conference Series in Mathematics, vol. 82, Published for the Conference Board of the Mathematical Sciences, Washington, DC, 1993.

[NVOo4] C. Năstăsescu and F. Van Oystaeyen, Methods of graded rings, Lecture Notes in Mathematics, vol. 1836, Springer-Verlag, Berlin, 2004.

[RZ15] L. Rigal and P. Zadunaisky, Twisted Semigroup Algebras, Alg. Rep. Theory 5 (2015), 1155-1186.

[PP12] A. Polishchuk and L. Positselski, Hochschild (co)homology of the second kind I, Trans. Amer. Math. Soc. 364 (2012), no. 10, 5311-5368.

[vdB97] M. van den Bergh, Existence theorems for dualizing complexes over non-commutative graded and filtered rings, J. Algebra 195 (1997), no. 2, 662-679.

[WZo3] Q.-S. Wu and J. J. Zhang, Applications of dualizing complexes, Proceedings of the Third International Algebra Conference (Tainan, 2002), Kluwer Acad. Publ., Dordrecht, 2003, pp. 241-255.

[Yek92] A. Yekutieli, Dualizing complexes over noncommutative graded algebras, J. Algebra 153 (1992), no. 1, 41-84.

[Yek14] A. Yekutieli, Another proof of a theorem of Van den Bergh about graded-injective modules (2014). Available at http://arxiv.org/abs/1407.5916

[YZ99] A. Yekutieli and J. J. Zhang, Rings with Auslander dualizing complexes, J. Algebra 213 (1999), no. 1, $1-51$.

[YZo9] _ Rigid dualizing complexes over commutative rings, Algebr. Represent. Theory 12 (2009), no. $1,19-52$.

[Zad14] P. Zadunaisky, Homological regularity properties of quantum flag varieties and related algebras, 2014. $\mathrm{PhD}$ Thesis. Available online at http://cms.dm.uba.ar/academico/carreras/doctorado/ desde.

A.S.:

IMAS-CONICET y Departamento de Matemática

Facultad de Ciencias Exactas y Naturales,

Universidad de Buenos Aires,

Ciudad Universitaria, Pabellón 1

1428, Buenos Aires, Argentina.

asolotar@dm.uba.ar 
P.Z. :

Instituto de Matemática e Estatística, Universidade de São Paulo.

Rua do Matão, 1010

CEP 05508-09o - São Paulo - SP

pzadun@ime.usp.br 